\title{
e-VLBI observations of a microquasar in outburst *
}

\author{
V. Tudose ${ }^{\dagger},{ }^{a b}$ R.P. Fender, ${ }^{c a}$ M.A. Garrett, ${ }^{d}$ J.C.A. Miller-Jones, ${ }^{a}$ Z. Paragi, ${ }^{d}$ R.E. \\ Spencer, ${ }^{e}$ G.G. Pooley, ${ }^{f}$ M. van der Klis, ${ }^{a}$ A. Szomoru ${ }^{d}$
}

'Astronomical Institute 'Anton Pannekoek', University of Amsterdam

${ }^{b}$ Astronomical Institute of the Romanian Academy

${ }^{c}$ School of Physics and Astronomy, University of Southampton

${ }^{d}$ Joint Institute for VLBI in Europe

${ }^{e}$ University of Manchester, Jodrell Bank Observatory

${ }^{f}$ University of Cambridge, Mullard Radio Astronomy Observatory

E-mail: vtudosedscience.uva.nl

We report the results of the first two 5-GHz e-VLBI observations of the X-ray binary Cygnus X-3 using the European VLBI Network. Two successful observing sessions were held, on 20 April 2006, when the system was in a quasi-quiescent state several weeks after a major flare, and on 18 May 2006, a few days after another flare. At the first epoch, we detected faint emission, probably associated with a fading jet, spatially separated from the X-ray binary. In contrast, the second epoch observation revealed a bright, curved, relativistic jet more than 40 milliarcseconds in extent. In the first, and probably also second epoch, the X-ray binary core was not detected, which may indicate a temporary suppression of jet production as seen in some black hole X-ray binaries in certain X-ray states. Polarisation measurements at the second epoch provide evidence of interaction between the ejecta and the surrounding medium. These results clearly demonstrate the importance of rapid analysis of long-baseline observations of transients, such as facilitated by e-VLBI.

8th European VLBI Network Symposium

September 26-29, 2006

Toruń, Poland

${ }^{*}$ This contribution is based on the paper 'First e-VLBI observations of Cygnus X-3' by Tudose et al., accepted as a letter to MNRAS.

† Speaker. 


\section{Introduction}

The X-ray binary Cygnus X-3 was first detected in X-rays at the end of the '60s [1]. Its infrared and X-ray fluxes show a periodicity of 4.8 hours, being interpreted as the orbital period of the system. The nature of the compact object is not known (e.g. [2] ), whereas the companion seems to be a WN Wolf-Rayet star (e.g. [3]). Radio observations at different resolutions were made during large flares, revealing the presence of relativistic jets in the system (e.g. [甘․

\section{Observations}

One of the aims of e-VLBI is to enable mapping of transient phenomena such as microquasars with long-baseline networks of radio telescopes in near real-time. In the case of the EVN, the data are transferred to the correlator at JIVE (Joint Institute for VLBI in Europe) in real-time using IP-routed networks, connecting the radio telescopes through national research networks (NRENs) and the pan-European research network GÉANT 2 via the Dutch NREN (SURFnet) to JIVE. Description of some of the development work in this field was given by [7] 8] and the most recent developments are presented in [7].

We observed Cygnus X-3 at $5 \mathrm{GHz}$ in phase-referencing mode in three sessions, on 16 Mar, 20 Apr and 18 May 2006 using the six antennas forming the current e-EVN: Onsala 20 m, Torun, Jodrell Bank MkII, Cambridge, Medicina and Westerbork (phased array). The observing run on 16 Mar was made in response to the first open call for e-EVN observations made two weeks earlier. Unfortunately, due to technical problems with the off-line correlator software, the data of 16 Mar were not usable and have been excluded from the present study. In our second e-VLBI session, on 20 Apr (epoch I), we observed Cygnus X-3 (in a 'quiescent' state) for an effective integration time of about 7 hours in a complex experiment consisting of interleaved observations of Cygnus X-3 and GRS 1915+105 [10]. At the beginning of May, Cygnus X-3 underwent a significant flare and we observed it during that active state via a target-of-opportunity proposal for about 12 hours on 18 May (epoch II).

The data were calibrated in AIPS using standard procedures (e.g. [11]). Self-calibration was applied once for phase with a 30-s solution interval and once for both amplitude and phase with a 30-min solution interval. The resulting radio maps are presented in Fig. 1.

\section{Results}

The maps shown in Fig. 1 account for a small fraction of the total flux density of the source: the integrated flux densities measured in the image plane are considerably lower than the ones obtained by fitting the data in the $u v$-plane with elliptical Gaussians. The reason for these differences is that because of the lack of $u v$-coverage at short baselines the large-scale emission could not be imaged properly. It is also worth mentioning that based on the simultaneous measurement of integrated flux density made with Westerbork at epoch II, we are missing almost 40 per cent of the emission at the milliarcsecond scale due to the lack of very short baselines in the e-EVN. 


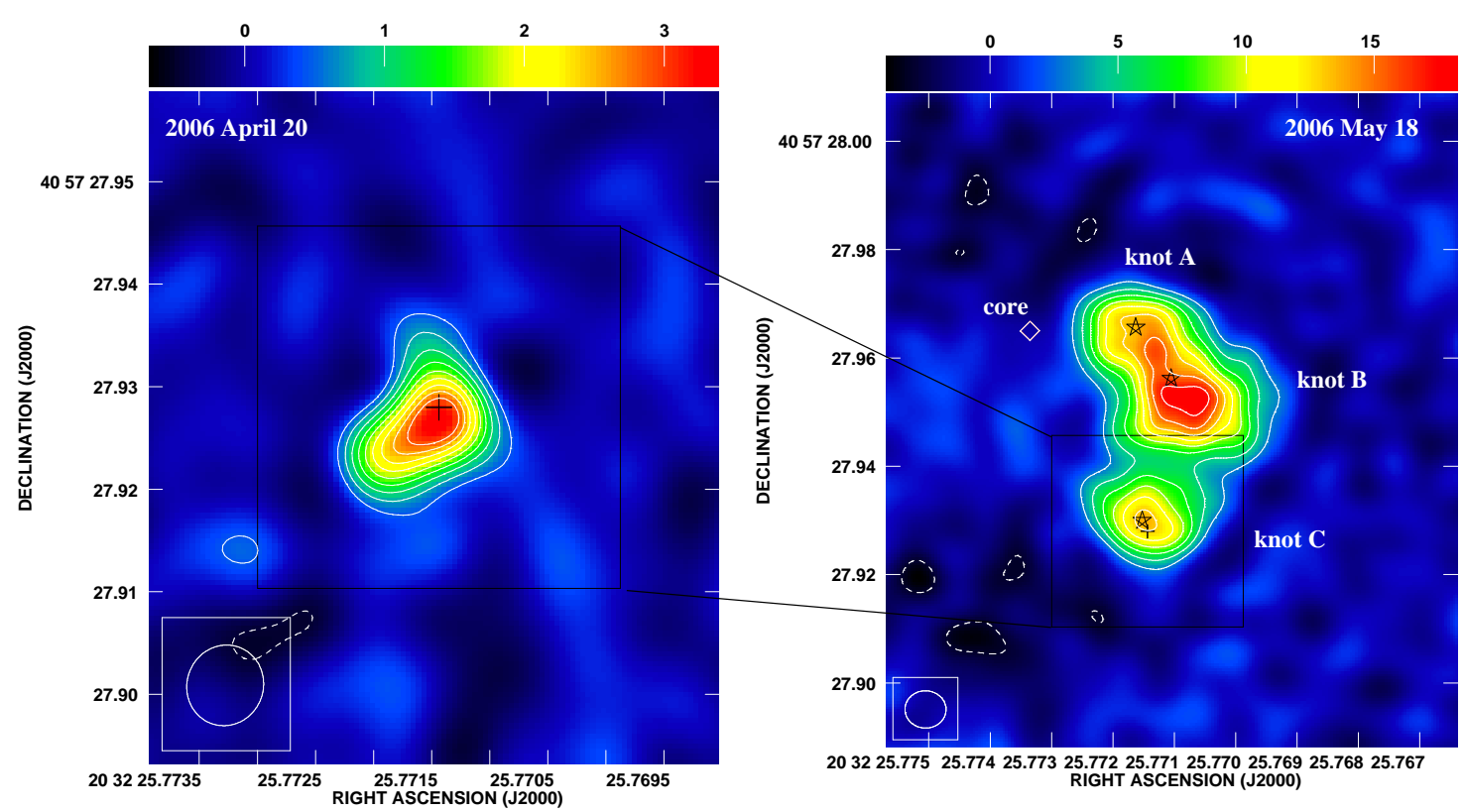

Figure 1: Left: The 5-GHz e-VLBI radio map of Cygnus X-3 of 20 Apr 2006 (epoch I). The contours are at $-15,15,25,35,45,55,65,75,85$, and $95 \times$ the rms noise of $0.03 \mathrm{mJy}^{\text {beam }^{-1}}$. The beam size is 7.9 $\times 7.4$ mas $^{2}$ at $\mathrm{PA}=-19.6$. The colour code bar on top of the map is expressed in mJy beam ${ }^{-1}$. Right: The 5-GHz e-VLBI radio map of Cygnus X-3 of 18 May 2006 (epoch II). The contours are at -15, 15, 25, $35,45,55,65,75,85$, and $95 \times$ the rms noise of $0.2 \mathrm{mJy}$ beam $^{-1}$. The beam size is $7.6 \times 6.9$ mas $^{2}$ at $\mathrm{PA}=88^{\circ} .3$. The colour code bar on top of the map is expressed in $\mathrm{mJy}$ beam $^{-1}$. The positions of the centres of the elliptical Gaussians fitted to the data are represented by a cross for epoch I and stars for epoch II. The estimated location of the radio core: $\mathrm{RA}=20^{\mathrm{h}} 32^{\mathrm{m}} 25 \mathrm{~s} 77335$, Dec $=40^{\circ} 57^{\prime} 27^{\prime \prime} \cdot 9650$ (J2000) [6] is indicated with a diamond. Note the different scales of the two images.

The single radio-emitting component observed at epoch I and knot " $\mathrm{C}$ " seen at epoch II are colocated within uncertainties. The large positional discrepancy with the previously estimated core position, plus the high degree of linear polarisation in the region ( $\sim 10$ per cent) suggests that knot "C" is probably not the core. Therefore, instead of the core, epoch I map likely shows an extended radio emission component physically separate from the X-ray binary that may be associated with previous flaring events. This in turn implies that the core itself was below detection levels with a $3 \sigma$ upper limit of $0.4 \mathrm{mJy}$, about two orders of magnitude fainter than typical levels for the source, and four orders of magnitude fainter than the brightest flares.

Epoch II reveals a much brighter and more complex structure: a curved jet with three bright knots. Knot " $\mathrm{A}$ " is clearly much closer to the previously estimated core location, but is still 24 mas away. In [ $₫$ ] and [6], the authors report the same core position to within 1 mas in the data separated by 4.5 years (but note that this is rather uncertain given the fact that [4] reported that their phasereferencing was not entirely successful). Based on the present knowledge, we cannot confidently identify the proper motion of the system as the cause of the shift. It seems likely therefore that even knot "A" may not correspond to the core, but simply to the nearest knot to it, indicating that at this epoch the core was also quenched. The orientation of the knots with respect to the core implies that all three are associated with the same side of the jet (likely to be approaching, although the 
complex environment of Cygnus X-3 may strongly influence appearances). Associating knot "C" with the large radio flare that occurred about a week earlier would imply proper motions in the jet of order 10 mas d $^{-1}$, consistent with those reported in [6]. For a distance of $\sim 9 \mathrm{kpc}$ [12], this corresponds to a projected velocity of $\sim 0.5 c$, and an intrinsic jet velocity at least that large.

In epoch II, the integrated flux densities measured using WSRT (at $5 \mathrm{GHz}$ ) and the Ryle Telescope (at $15 \mathrm{GHz}$ ) reveal a steep spectrum $\left(\propto v^{-0.6}\right)$, indicative of optically thin synchrotron emission. However, since the map presented in Fig. 1 recovers $\leq 15$ per cent of the integrated flux density, we cannot definitively draw the conclusion that the imaged structures are therefore also optically thin synchrotron emission. Nevertheless, based on simple physical arguments and comparison with other relativistic jet sources, this is likely, and is consistent with the relatively high levels of polarisation observed along the jet. As noted above, knot "C" in particular shows a high level of polarisation, which supports the interpretation that it is not the core. The highest fractional polarisation, almost 25 per cent, appears east of knot " $\mathrm{B}$ ". This is suggestive of an interaction site between the ejected matter and the surrounding medium (possibly the material ejected in a previous active state). The coincidence of emission at the location of knot " $\mathrm{C}$ " in both epochs may suggest that this is the location of a standing shock in the flow or a site of repeated jet-ISM interactions.

\section{Conclusions}

We have presented results on the X-ray binary Cygnus X-3 obtained in the framework of the first open call for e-EVN observations. Our two successful experiments captured Cygnus X-3 initially in an almost quiescent state after a previous outburst, and subsequently in an active state a few days after the major flare in May 2006. In the second epoch, a bright, curved, relativistic jet was observed. The total intensity radio maps and polarisation measurements provide evidence for interaction of the jet with the surrounding medium and, if our interpretation is correct, with the material ejected in the previous active state. In the first epoch, two weeks prior to the onset of the major flare of May 2006, the core was in a deeply radio-quenched state, with a $3 \sigma$ upper limit to the flux density of $0.4 \mathrm{mJy}$. The use of e-VLBI enabled very quick access to the data, practically within one day after the end of the runs, compared to weeks in the traditional VLBI experiments. This possibility for rapid response and analysis of VLBI data is of paramount importance for the study of transient sources, enabling decision making with respect to potential follow-up observations (not only at radio wavelengths) to be much more effective.

\section{Acknowledgments}

We thank the JIVE staff R.M. Campbell and C. Reynolds for their valuable contribution to the technical success of the experiments. The European VLBI Network is a joint facility of European, Chinese, South African and other radio astronomy institutes funded by their national research councils. The Ryle Telescope is operated by the University of Cambridge. The X-ray data were provided by the ASM/RXTE teams at MIT and at the RXTE SOF and GOF at NASA's GSFC. e-VLBI developments in Europe are supported by the EC DG-INFSO funded Communication Network Development project, 'EXPReS', Contract No. 02662. 


\section{References}

[1] Giacconi R., Gorenstein P., Gursky H., Waters J.R., 1967, ApJ, 148, L119

[2] Mitra A., 1998, ApJ, 499, 385

[3] Koch-Miramond L., Ábrahám P., Fuchs Y., Bonnet-Bidaud J.-M., Claret A., 2002, A\&A, 396, 877

[4] Mioduszewski A.J., Rupen M.P., Hjellming R.M., Pooley G.G., Waltman E.B., 2001, ApJ, 553, 766

[5] Martí J., Paredes J.M., Peracaula M., 2001, A\&A, 375, 476

[6] Miller-Jones J.C.A., Blundell K.M., Rupen M.P., Mioduszewski A.J., Duffy P., Beasley A.J., 2004, ApJ, 600, 368

[7] Parsley S., Pogrebenko S., Szomoru A., Verkouter H., Burgess P., Hughes-Jones R., Spencer R., Mujunen A., Ritakari J., 2003, in ASP Conf. Ser., 306, New technologies in VLBI, ed. Y.C. Minh

[8] Szomoru A., Biggs A., Garrett M., van Langevelde H.J., Olnon F., Paragi Z., Parsley S., Pogrebenko S., Reynolds C., 2004, in Proceedings of the 7th Symposium of the European VLBI Network on New Developments in VLBI Science and Technology, eds. R. Bachiller, F. Colomer, J.-F. Desmurs and P. de Vicente

[9] Szomoru A., 2006, in Proceedings of the 8th European VLBI Network Symposium, eds. A. Marecki et al., $\operatorname{PoS}(8 \mathrm{thEVN}) 053$

[10] Rushton A., Spencer R.E., Strong M., Campbell R.M., Casey S., Fender R.P., Garrett M.A., Miller-Jones J.C.A., Pooley G.G., Reynolds C., Szomoru A., Tudose V., Paragi Z., 2006, in Proceedings of the 8th European VLBI Network Symposium, eds. A. Marecki et al., $\operatorname{PoS}(8$ thEVN $) 081$

[11] Diamond P.J., 1995, in ASP Conf. Ser. 82, Very Long Baseline Interferometry and the VLBA, eds. J.A. Zensus, P.J. Diamond and P.J. Napier

[12] Predehl P., Burwitz V., Paerels F., Trümper J., 2000, A\&A, 357, L25 